\title{
Symbolic derivation of bicycle kinematics with toroidal wheels
}

\author{
Everett X. Wang ${ }^{1, a}$, Juncheng Zou ${ }^{1, b}$, Gengping Xue ${ }^{1, \mathrm{c}}$,Lin Yuan ${ }^{1, \mathrm{~d}}$, Zhaoheng Zeng ${ }^{1, \mathrm{e}}$, Gengfeng Xue $^{1, \mathrm{f}}$, Yijun Liu $^{1, \mathrm{~g}}$, \\ Gary Zhang ${ }^{1, \mathrm{~h}}$ and Qun Fan ${ }^{1, \mathrm{i}}$
}

No. 100 Waihuan Xi Road, Guangdong University of Technology, Guangzhou HEMC,

Panyu District, Guangzhou, Guangdong Province, China, 510006

\begin{abstract}
Bicycle kinematics with toroidal wheels is presented in this paper. Using symbolic mathematic tool Maple, we obtain two holonomic and four nonholonomic constraint equations due to front and rear wheels of a bicycle. We show that the two holonomic constraints cannot be expressed in quartic form for bicycle rear body pitch angle unless the minor (crown) radius of the torus are the same for both the front and rear wheels. In addition, we show that all the constraints can be written in differential form, from which a constraint matrix is constructed, according to standard procedure for developing dynamics in robotics.
\end{abstract}

\section{Introduction}

Recent progress in autonomous vehicles from various motor companies including Audi, General Motor, Mercedes-Benz, Toyota as well as Google grabs headlines [1], provoking further researches in vehicle sensing and control. The self-driving vehicles utilize the knowledge developed in robotics for sensing, dynamic modeling and control to perform various activities normally requiring a driver to do. Robotic single-track vehicles such as motorcycle and bicycle have potential to become the most energy efficient with small foot-print for high performance urban transportation. However, it faces more challenges due to the fact that it is unstable statically and elaborated dynamic controls have to be developed to make it self-balancing while following a defined path.

Most modern control schemes require an accurate dynamic model for the vehicle. Even though bicycle can be approximated as four interconnected rigid bodies, its dynamics is surprisingly complex. Even today, there are still many unresolved questions related to bicycle dynamic behaviors such as mechanisms of self-stability [2] and nonlinear controllability. Bicycle dynamics has been studied extensively over the years. Most of the papers have been published trying to deal with linear and nonlinear models (see the extensive literature review in [3]). The most successful bicycle dynamic model is the Whipple-Cornell-Delft benchmark bicycle [3], which has explained linear motion of bicycle steer and roll dynamics very well. More importantly it serves as a benchmark and allows many models, which use different coordinate systems with different assumptions, to be compared. Work has been done to extend the model to include more physics and make it nonlinear so its application range can be expanded. J. P. Meijaard et al. extended the linear model to include more realistic tires [4], while Fraseli et al. constructed bicycle kinematics with toroidal wheels instead of knife-edge wheels [5]. Both [4,5] derived their complex results using pencil and paper. Wang et al. used symbolic mathematics to extended Whipple-Cornell-Delft bicycle into a nonlinear dynamic model [6]. Symbolic tools reduce possible human errors during calculations. In this paper we apply symbolic tool Maple to include the toroidal wheels into kinematics for the benchmark bicycle.

The paper is organized as follows. We first present our general coordinate systems for bicycle with toroidal wheels and show that nine coordinates are needed to describing bicycle's dynamic state in section 2 . In section 3 we derive two holonomic constraints due to the requirements that the front wheel and rear wheel touching the road surface. A Newton iterative scheme is applied to solve the rear body pitch angle from the two constraints. Then four nonholonomic constraints are derived from front and rear wheel nonslip conditions in section 4. Finally we summarize our results in section 5 .

\section{General coordinates for bicycle with toroidal wheels}

The Whipple-Cornel-Delft benchmark model is composed of four rigid bodies: the rear vehicle body (B) which also includes a rider rigidly attached to it, rear wheel (R), Handlebar/fork $(\mathrm{H})$ and front wheel $(\mathrm{F})$. It has a realistic geometrics as well as arbitrary mass distribution and has been used extensively for study bicycle dynamics. To extend the toroidal wheels in the Whipple-Cornel-Delft benchmark bicycle one mainly has to modify constraint

${ }^{\mathrm{a}}$ Everett X. Wang: everett.wang@gdut.edu.cn 
equations due to the tires non-slipping, non-penetrating on the plane road surface. Fig. 1 shows the 4 rigid bodies of the bicycle and their body-fixed coordinate frames.

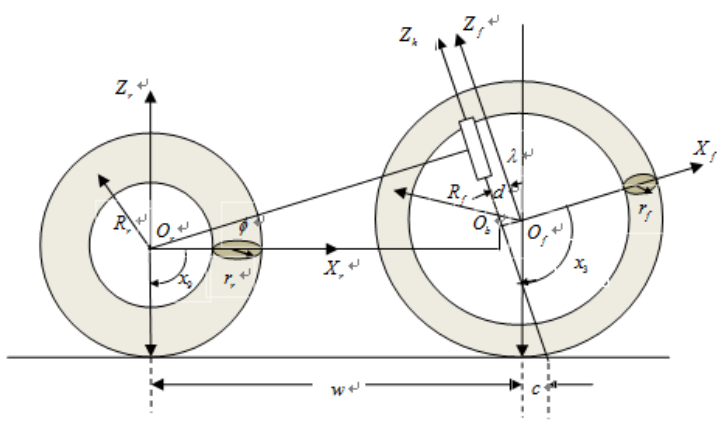

Fig 1. Body-fixed frames and bicycle geometry with toroidal wheels.

We adopted bicycle parameters from the extended linear model in [4]. Their geometric parameters are listed in Table 1. Note that the front wheel center offset $d=\left(R_{f}+r_{f}\right) \sin \lambda-c \cdot \cos \lambda[5]$.

Table 1. Geometric parameters for the bicycle

\begin{tabular}{|c|c|c|}
\hline Symbol & Meaning & Value \\
\hline $\mathrm{w}$ & wheelbase & $1.02(\mathrm{~m})$ \\
\hline $\mathrm{c}$ & trail & $0.08(\mathrm{~m})$ \\
\hline$\lambda$ & $\begin{array}{c}\text { caster } \\
\text { angle }\end{array}$ & $0.1 \pi(\mathrm{rad})$ \\
\hline$R_{r}$ & $\begin{array}{c}\text { rear wheel } \\
\text { radius }\end{array}$ & $0.3(\mathrm{~m})$ \\
\hline$r_{r}$ & $\begin{array}{c}\text { rear wheel } \\
\text { crown radius }\end{array}$ & $0.02(\mathrm{~m})$ \\
\hline$R_{f}$ & $\begin{array}{c}\text { front wheel } \\
\text { radius }\end{array}$ & $0.35(\mathrm{~m})$ \\
\hline$r_{f}$ & $\begin{array}{c}\text { front wheel } \\
\text { crown radius }\end{array}$ & $0.015(\mathrm{~m})$ \\
\hline
\end{tabular}

Using the same general coordinates systems in [6], we can describe the motion of each body using a $4 \times 4$ matrix, relating the body fixed coordinates to an inertial frame. A point in rear body frame with its body-fixed coordinate of $\left[\begin{array}{llll}x_{b} & y_{b} & z_{b} & 1\end{array}\right]^{T}$, for example, corresponds to coordinate $\left[\begin{array}{llll}x & y & z & 1\end{array}\right]^{T}$ in the inertial frame through a rotation and a translation described by matrix $T_{b}$ :

$$
\left[\begin{array}{c}
x \\
y \\
z \\
1
\end{array}\right]=T_{b}\left[\begin{array}{c}
x_{b} \\
y_{b} \\
z_{b} \\
1
\end{array}\right]
$$

Each of the 4 body has its own body-fixed frame (see Fig. 1) and a 4 by 4 matrix relating the body-fixed coordinate to the inertial frame. The matrices $T_{b}, T_{r}, T_{h}, T_{f}$, for rear vehicle body (b), rear wheel (r), handlebar/fork (h) and front wheel (f) respectively, depend on nine general coordinates, $x_{1} \ldots x_{9}$. Their meaning and sign conventions are listed in Table 2. The state of the vehicle is fully described by the general coordinates through the 4 matrices. Their expressions can be found in [6]. Due to the six constraints, the model has only three degrees of freedom (DOF). We select $x_{7}, x_{8}, x_{9}$ to represent the DOF.
Table 2. Names, meanings and sign conventions of generalized coordinates of the bicycle model

\begin{tabular}{|c|c|c|}
\hline $\begin{array}{l}\text { Variable } \\
\text { name }\end{array}$ & Meaning & Note \\
\hline$x_{1}$ & $\begin{array}{l}\text { Rear body } \\
\text { pitch }\end{array}$ & $\begin{array}{l}\text { Front of vehicle } \\
\text { lift up is negative }\end{array}$ \\
\hline$x_{2}$ & $\begin{array}{l}\text { Rear body } \\
\text { yaw }\end{array}$ & $\begin{array}{c}\text { Deviation from } \\
\text { positive } \mathrm{x} \text { counter } \\
\text { clock wise is positive }\end{array}$ \\
\hline$x_{3}$ & $\begin{array}{c}\text { Front } \\
\text { wheel rotation } \\
\text { angle } \\
\end{array}$ & $\begin{array}{l}\text { Going forward is } \\
\text { positive }\end{array}$ \\
\hline$x_{4}$ & $\mathrm{x}$ of $O_{b}$ & \multirow{3}{*}{$\begin{array}{c}\text { Original of } \\
o_{b} \text { measured in an } \\
\text { inertial frame }\end{array}$} \\
\hline$x_{5}$ & y of $O_{b}$ & \\
\hline$x_{6}$ & $\mathrm{z}$ of $O_{b}$ & \\
\hline$x_{7}\left(q_{1}\right)$ & $\begin{array}{l}\text { Rear body } \\
\text { roll }\end{array}$ & $\begin{array}{l}\text { Lean to the right } \\
\text { is positive }\end{array}$ \\
\hline$x_{8}\left(q_{2}\right)$ & Steer angle & $\begin{array}{l}\text { Turn to the left is } \\
\text { positive }\end{array}$ \\
\hline$x_{9}\left(q_{3}\right)$ & $\begin{array}{c}\text { Rear wheel } \\
\text { rotation angle }\end{array}$ & $\begin{array}{l}\text { Going forward is } \\
\text { positive }\end{array}$ \\
\hline
\end{tabular}

\section{Holonomic constrains and rear body pitch}

The holonomic constrains are due to the fact that both front and rear wheels are required to touch the road surface. Let's start with rear toroidal wheel. Normally a torus can be parameterized by two angles, one forming a circle with a minor (crown) radius, while the other rotating the circle to form the torus with a major (wheel) radius. Here we picked $\phi$ to form the minor circle with radius $r_{r}$ in its body fixed frame (Fig. 1). The tire surface in the inertial frame is then:

$$
\left[\begin{array}{c}
x \\
y \\
z \\
1
\end{array}\right]=T_{r}\left[\begin{array}{c}
R_{r}+r_{r} \cos \phi \\
-r_{r} \sin \phi \\
0 \\
1
\end{array}\right]
$$

When the parameter $\phi$ and $x_{9}$ in $T_{r}$ vary from 0 to $2 \pi$, $\left[\begin{array}{llll}x & y & z & 1\end{array}\right]^{T}$ forms a complete torus with crown radius $r_{r}$ and wheel radius of $R_{r}$ in the inertial frame. The rear wheel holonomic constrain is to require the lowest point of the surface to be zero height, which can be located by setting the derivatives of the height vs. $x_{9}$ and $\phi$ to zero. Symbolic calculation shows the height as function of general coordinates and parameters $x_{9}$ and $\phi$ :

$H_{r}=z=w \cdot s x_{1}+d \cdot\left(c x_{1} c x_{7} \sin \lambda-s x_{1} \cos \lambda\right)-s x_{1}\left(R_{r}+r_{r} \cos \phi\right) \cos x_{9}$ $-r_{r} c x_{1} s x_{7} \sin \phi+\left(R_{r}-R_{f}\right) c x_{1} c x_{7}-\left(R_{r}+r_{r} \cos \phi\right) c x_{1} c x_{7} \sin x_{9}+x_{6}$

$$
\frac{\partial H_{r}}{\partial x_{9}}=0, \frac{\partial H_{r}}{\partial \phi}=0
$$

Solving the two above equations, we can readily find the $x_{9}$ and $\phi$ at the contact point: $x_{9 r c}$ and $\phi_{r c}$ (rc for rear contact). They are functions of $x_{1}, x_{7}$. Plugging in their solutions into the expression for the height and setting it to zero, we now have our rear wheel holonomic constraint as: 


$$
\begin{aligned}
& H_{r}=w \cdot s x_{1}-d \cdot\left(s x_{1} \cos \lambda-c x_{1} c x_{7} \sin \lambda\right)+\left(R_{r}-R_{f}\right) c x_{1} c x_{7} \\
& -R_{r} \sqrt{c x_{1}^{2} c x_{7}^{2}+s x_{1}^{2}}+x_{6}-r_{r}=0
\end{aligned}
$$

where we denote $s x_{1}=\sin \left(x_{1}\right), c x_{1}=\cos \left(x_{1}\right)$, etc for simplicity. The front wheel holonomic constrain can be derived similarly:

$$
H_{f}=d \cdot a_{2}-R_{f} \sqrt{a_{1}^{2}+a_{2}^{2}}+x_{6}-r_{f}=0
$$

where $a_{1}$ and $a_{2}$ are functions of general coordinates:

$$
\begin{aligned}
& a_{1}=-s x_{1} \cdot \sin \lambda-c x_{1} \cdot c x_{7} \cdot \cos \lambda \\
& a_{2}=-\cos \lambda s x_{1} c x_{8}+c x_{1} s x_{7} s x_{8}+\sin \lambda c x_{1} c x_{7} c x_{8}
\end{aligned}
$$

Subtracting eq. 2 and eq. 3, we can eliminate $x_{6}$, leaving an equation contains only general coordinates $x_{1}, x_{7}, x_{8}$. It can be used to solve $x_{1}$, the rear body pitch angle and express it in $x_{7}, x_{8}$, the rear body roll angle and steer angle, respectively. Unlike the knife-edge wheel case, where the equation contains two square root terms and can be converted into a quartic form, the pitch equation with toroidal wheels cannot be converted into a quartic form hence analytic solution doesn't exist unless front and rear wheel crown radii $r_{f}, r_{r}$ are the same. Fig. 2 depicts the numerical solution using a Newton iterative scheme.

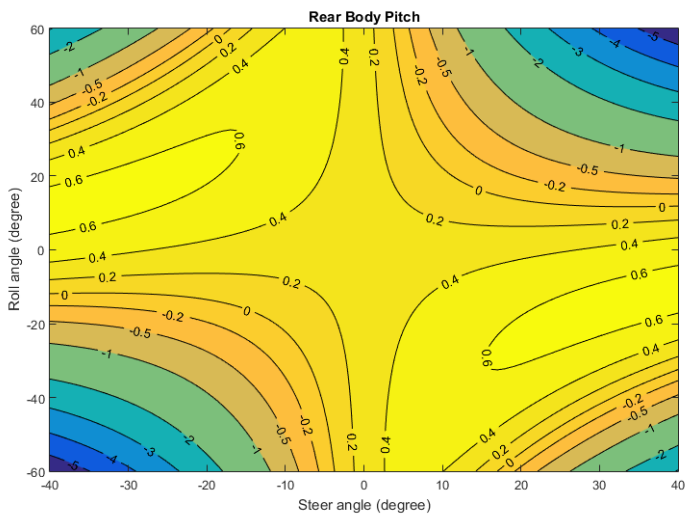

Fig 2. Pitch angle of rear vehicle body in degree vs. steer and roll angle. The pitch angle is solved using Newton iterative scheme.

\section{Nonholonomic constraints}

Unlike holonomic constraints, which can be written as $f(x)=0$, nonholonomic constraints can only be expressed in differential form. The nonholonomic constraints are the results from the requirements that the toroidal wheels only roll on the road surface without slip in longitudinal and transversal directions. Taking time derivation in Eq. 1, we can find velocity vector on the tire surface:

$$
\vec{v}_{r}=\left[\begin{array}{c}
\dot{x}_{r} \\
\dot{y}_{r} \\
\dot{z}_{r} \\
0
\end{array}\right]=\dot{T}_{r}\left[\begin{array}{c}
R_{r}+r_{r} \cos \phi \\
-r_{r} \sin \phi \\
0 \\
1
\end{array}\right]
$$

Setting the $\mathrm{x}$ and $\mathrm{y}$ velocity to zero at the tire-road surface contact point we have the two nonholonomic constraints from the rear wheel as:

$$
\begin{gathered}
\left.\dot{x}_{r}\right|_{x_{9}=x_{9 r c}, \phi=\phi_{r c}}=a_{11} \dot{x}_{1}+a_{12} \dot{x}_{2}+\dot{x}_{4}+a_{17} \dot{x}_{7}+a_{19} \dot{x}_{9}=0 \\
\left.\dot{y}_{r}\right|_{x_{9}=x_{9 r c}, \phi=\phi_{r c}}=a_{21} \dot{x}_{1}+a_{22} \dot{x}_{2}+\dot{x}_{5}+a_{27} \dot{x}_{7}+a_{29} \dot{x}_{9}=0
\end{gathered}
$$

Similarly front wheel nonholonomic constraints can be obtained. They have the similar differential form:

$$
\begin{aligned}
& \left.\dot{x}_{f}\right|_{x_{3}=x_{3 \mathfrak{f c}}, \phi=\phi_{f c}}=a_{31} \dot{x}_{1}+a_{32} \dot{x}_{2}+a_{33} \dot{x}_{3}+\dot{x}_{4}+a_{37} \dot{x}_{7}+a_{38} \dot{x}_{8}=0 \text { (7) } \\
& \left.\dot{y}_{f}\right|_{x_{3}=x_{3 \mathfrak{c}}, \phi=\phi_{f c}}=a_{41} \dot{x}_{1}+a_{42} \dot{x}_{2}+a_{43} \dot{x}_{3}+\dot{x}_{5}+a_{47} \dot{x}_{7}+a_{48} \dot{x}_{8}=0
\end{aligned}
$$

where $x_{3 f_{c}}, \vartheta_{f_{c}}$ are front wheel rotation angle and camber angle at the wheel-road surface contact point. Taking a time derivation on the Eq. 2 and 3 we can also write the holonomic constraints into differential forms:

$$
\begin{gathered}
a_{51} \dot{x}_{1}+\dot{x}_{6}+a_{57} \dot{x}_{7}=0 \\
a_{61} \dot{x}_{1}+\dot{x}_{6}+a_{67} \dot{x}_{7}+a_{68} \dot{x}_{8}=0
\end{gathered}
$$

Eq. 5 through 10 are the six constrain equations for bicycle with toroidal wheels. They can be combined into a nice matrix format:

$$
A(x) \dot{x}=0,
$$

where $A(x)$ is a 6 by 9 matrix. All the elements are analytic functions of $x_{1}, x_{2}, x_{7}, x_{8}$. Due to the page limitation, they will not be included here. Interesting readers can contact authors for Maple and Matlab scripts that produce all the symbolic and numerical results in this paper.

\section{Summary}

We have presented a bicycle kinematics with toroidal wheels in this paper. Using symbolic mathematic tool Maple, we proved that the two holonomic constraint equations can be used to obtain vehicle rear body pitch. Unless the front and rear tire crown radii are the same, the pitch equation cannot be written in quartic form, preventing us from obtaining analytic solution. Moreover, we have shown that all the constraints can be written in differential form, suitable for developing nonlinear dynamics. We plan to use the kinematics developed in this paper to build a nonlinear bicycle dynamic model with toroidal wheels.

\section{References}

1. "Google's Self-Driving Cars: 300,000 Miles Logged, Not a Single Accident Under Computer Control", The Atlantic, Retrieved on April 20, 2015

2. J. D. G. Kooijman, J. P. Meijaad, Jim M. Papadopoulos, Andy Ruina and A. L. Schwab, "A Bicycle can be self-stable without gyroscopic or caster effects", Science, 332, 2011, p. 339

3. J. P. Meijaard, J. M. Papadopoulos, A. Ruina, and A. L. Schwab, "Linearized dynamics equations for the 
balance and steer of a bicycle: a benchmark and review”. Proc. R. Soc. A 463, 2007, pp. 1955-1982,

4. J. P. Meijaard and A. L. Schwab, "Linearized equation for an extended bicycle model", III European Conference on Computational Mechanics, Solids, Structures and Coupled Problems in Engineering, Lisbon, Portugal, 5-9 June 2006.

5. G. Frosali, F. Ricci, "Kinematics of a bicycle with toroidal wheels", Communications in Applied and Industrial Mathematics, 3(1) (2012) DOI: 10.1685/journal.caim.424

6. Everett X. Wang, Juncheng Zou, Gengping Xue, Yijun Liu, Yang Li and Qun Fan, "Development of efficient nonlinear benchmark bicycle dynamics for control applications", IEEE Transaction on Intelligent Transportation Systems, Vol. 16, No. 4, Aug. 2015, pp. 2236-2246. 